\section{Dr. Schlesinger replies}

\section{To the Editor:}

Risk factors and comorbidities associated with erectile dysfunction (ED) in patients with gout include diabetes mellitus, hypertension, advanced age, obesity, hyperlipidemia, metabolic syndrome, certain medications, and tobacco abuse. We suggested ${ }^{1}$ that hyperuricemia and inflammation may be independent risk factors for ED in addition to the conventional ones. Lv and Chen ${ }^{2}$ suggest another possible independent risk factor for ED in patients with gout: low levels of Vitamin D, and they question the need to give Vitamin D supplementation to these patients.

Vitamin D might have both positive and negative effects on the cardiovascular system and thus on ED, depending on the doses used. In 2007, Zittermann, $e a^{3}$ suggested a "U" curve to explain the potential dual effect of Vitamin D on the cardiovascular system - an increased cardiovascular risk at both the low (mainly secondary to the reduced antiinflammatory and endothelial-protective effects) and high (secondary to the increased calcemic and phosphatemic effects and stimulation of FGF23) Vitamin D levels. For the dual effect to be studied, the study duration should be sufficiently long; although the antiinflammatory effect might be evident in a short time, to see the effects related to calcium and phosphorus metabolism might require a longer observation time.

The evidence base for effects of Vitamin D on bone health is strong and supported by randomized clinical trials. However, the evidence base for the effects of Vitamin D on nonskeletal disease such as cardiovascular disease and ED is inconclusive, inconsistent, and unable to support such conclusions ${ }^{4}$. Thus, the question of the effect of Vitamin D levels as well as Vitamin D supplementation on serum urate and ED in patients with gout is interesting and may warrant further study.

NAOMI SCHLESINGER, MD, Rutgers-Robert Wood Johnson Medical School, MEB 468, P.O. Box 19, New Brunswick, New Jersey 08903, USA. Address correspondence to Dr. N. Schlesinger;

e-mail: schlesna@rutgers.edu

\section{REFERENCES}

1. Schlesinger N, Radvanski D, Cheng JQ, Kostis JB. Erectile dysfunction is common among patients with gout. J Rheumatol 2015;42:1893-7.

2. Lv ZT, Chen AM. Vitamin D deficiency may explain the possible link between gout and erectile dysfunction. J Rheumatol 2016;43:1617.

3. Zittermann A, Schleithoff SS, Koerfer R. Vitamin D and vascular calcification. Curr Opin Lipidol 2007;18:41-6.

4. Ross AC, Manson JE, Abrams SA, Aloia JF, Brannon PM, Clinton SK, et al. The 2011 report on dietary reference intakes for calcium and vitamin D from the Institute of Medicine: what clinicians need to know. J Clin Endocrinol Metab 2011;96:53-8.

J Rheumatol 2016;43:8; doi:10.3899/jrheum.160394 